\title{
Granular forces from steady and avalanche flows on a wall-like obstacle: contribution to avalanche dam design
}

\author{
T. Faug, B. Chanut \& M. Naaim \\ Cemagref, ETGR, St-Martin d'Hères, France
}

\begin{abstract}
The present paper deals with the hydrodynamic force from granular streams overflowing a wall-like obstacle. An analytical continuum model is proposed to derive this force both for steady and unsteady flow conditions. The force derived from the continuum model is successfully compared to the time-averaged force from discrete numerical simulations of free-surface granular flows down an incline and overflowing a wall. A good agreement is found in a large range of slope inclinations from a slow dense regime to a rapid dilute one. The continuum model gives the various contributions of the total force. Application to avalanche dam design is discussed.

Keywords: granular avalanche, protection dam, obstacle, force, continuum model, discrete simulations.
\end{abstract}

\section{Introduction}

Gravity-driven mass movements such as dense avalanches of granular snow can lead to huge damages on structures (buildings and protection dams). The flowobstacle interaction is a crucial question to better understand the influence of obstacles on flows of granular fluids and the force that granular fluids can exert on the obstacle. In this paper, an analytical continuum model is proposed to derive the force from avalanches overflowing a wall-like obstacle. The model is based on momentum and mass conservations applied to a control volume which corresponds to the upstream volume disturbed by the presence of the obstacle. Small-scale discrete numerical simulations on granular flows down an incline and meeting a wall were carried out to calibrate and test the continuum model. Steady 
recirculating flows and time-varying avalanche flows were studied. In section 2 are presented the continuum model equations. The section 3 briefly describes the discrete elements method (DEM), the simulated systems and the main results in terms of force on the wall (further details can be found in $[1,2,6]$ ). We focus our attention on the stagnant zone, formed upstream the obstacle, and inside which important fluctuations occur. The section 4 is devoted to the continuum model calibration (closure equations) and its prediction in term of force for both steady and transient flows. A good agreement between the time-averaged force from the DEM simulations and the continuum model force is found whatever the flow regime, from the slow dense regime at low slope inclinations to the rapid dilute regime at larger slopes. The section 5 concludes the paper about the interest and the limits of the proposed analytical continuum model for the design of structures in avalanche prone areas.

\section{Continuum model equations}

\subsection{Steady flows}

Let us define the control volume $V_{0}$ as the fluid volume disturbed by the presence of the wall-like obstacle upstream of the obstacle (see sketch in Fig. 1). The momentum conservation in the control volume $V_{0}$ is:

$$
\oint_{S} \rho \vec{u}(\vec{u} \cdot \vec{n}) d S=\oint_{S} p \vec{n} d S+\oint_{S} \sigma \cdot \vec{n} d S+\iint_{V_{0}} \vec{g} d V
$$

where $S$ is the boundary surface of the control volume $V_{0}: S=S_{1} \cup S_{2} \cup S_{b} \cup$ $S_{o} \cup S_{f s}$ with $S_{1}$ the incoming flow section, $S_{2}$ the outflow section, $S_{b}$ the surface at the base of the control volume (contact with the bottom), $S_{o}$ the surface of the wall-like obstacle and $S_{f s}$ the surface corresponding of the free-surface of the flow (contact with the air). The flow is $2 \mathrm{D}$, the forces are expressed by unit width (in N.m $\mathrm{m}^{-1}$ ). $\vec{n}$ is the unit vector normal to the surface $S, \rho$ is the flow density ( $\rho=\rho_{P} \phi$ where $\rho_{P}$ is the particle density and $\phi$ the volume fraction), $\vec{u}$ is the velocity vector, $\vec{g}$ the gravity acceleration, $p$ the pressure and $\sigma$ the stress tensor. With the mass conservation applied between sections $S_{1}$ and $S_{2}$, we obtain:

$$
\beta Q_{m}^{0}\left(\vec{u}_{e}-\vec{u}\right)=\vec{P}+\vec{F}_{0}+\vec{F}_{1}+\vec{F}_{2}+\vec{R}-\vec{F}
$$

where $\vec{u}$ and $\vec{u}_{e}$ are the depth-averaged velocities at sections $S_{1}$ and $S_{2}$ respectively and $Q_{m}^{0}=\bar{\rho} h \bar{u}$ is the mass flow rate per unit width. $P$ is the weight of the control volume, $F_{0}$ is the pressure force at the contact surface with the air $\left(S_{f s}\right), F_{1}$ is the pressure force in section $S_{1}, F_{2}$ is the pressure force in section $S_{2}, R$ is the reaction of the bottom and $F$ is the force on the obstacle. The assumptions made to derive these forces are discussed in detail in [6]. The projections of equation (2) on the $x$-axis direction (parallel to the bottom) and on the $y$-axis direction (normal to the bottom) give the resulting normal $\left(F_{N}\right)$ and tangential $\left(F_{T}\right)$ forces on the obstacle: 


$$
\begin{gathered}
F_{N}=\beta \bar{\rho} \bar{u}^{2} h\left(1-\delta_{u} \cos \alpha^{0}\right)+\frac{1}{2} k \bar{\rho} g h^{2} \cos \theta+\bar{\rho}_{0} V_{0} g \sin \theta-\bar{\mu}_{z m} R_{y} \\
F_{T}=\beta \bar{\rho} \bar{u}^{2} h \delta_{u} \sin \alpha^{0}-\bar{\rho}_{0} V_{0} g \cos \theta+R_{y}
\end{gathered}
$$

where $h$ is the incoming flow thickness, $\bar{u}$ its depth-averaged velocity and $\bar{\phi}$ its depth-averaged density. $\theta$ is the slope inclination. $\rho_{0}$ is the mean volume fraction in the control volume $V_{0} . \beta$ and $k$ are model parameters: $\beta$ is the coefficient related to the velocity profile across the layer $\left(\bar{u}^{2}=\beta \bar{u}^{2}\right)$ and $k$ is the ratio of the longitudinal normal stress $\sigma_{x x}$ to the vertical normal stress $\sigma_{y y} . \alpha^{0}$ is the deflection angle due to the obstacle (angle between $\vec{u}_{e}$ and the bottom) and $\delta_{u}=\bar{u}_{e} / \bar{u}$ defines the velocity reduction between sections $S_{1}$ and $S_{2} \cdot \bar{\mu}_{z m}$ is a space-averaged friction coefficient defining the component in the $x$-axis direction of the bottom reaction $\vec{R}$ as a basal coulombian force: $R_{x}=\bar{\mu}_{z m} R_{y}$, where $R_{y}$ is the component in the $y$-axis direction of the bottom reaction $\vec{R}$.

Combining equations (3) and (4), the normal force $F_{N}$ is:

$$
\begin{gathered}
F_{N}=F_{d}^{N}+F_{h}^{N}+F_{w}^{N}-\bar{\mu}_{z m}\left(F_{T}+F_{w}^{T}+F_{d}^{T}\right) \\
F_{d}^{N}=\beta \bar{\rho} \bar{u}^{2} h\left(1-\delta_{u} \cos \alpha^{0}\right) \\
F_{h}^{N}=\frac{1}{2} k \bar{\rho} g h^{2} \cos \theta \\
F_{w}^{N}=\bar{\rho}_{0} V_{0} g \sin \theta \\
F_{w}^{T}=\bar{\rho}_{0} V_{0} g \cos \theta \\
F_{d}^{T}=-\beta \bar{\rho} \bar{u}^{2} h \delta_{u} \sin \alpha^{0}
\end{gathered}
$$

Considering that the tangential force on the obstacle $\left(F_{T} \sim 0\right)$ is negligible (see DEM simulations in section 3.3), we obtain:

$$
F_{N}=F_{d}^{N}+F_{h}^{N}+F_{w}^{N}-\bar{\mu}_{z m}\left(F_{w}^{T}+F_{d}^{T}\right)
$$

A remarkable process is the formation of a stagnant zone upstream of the wall (see Fig. 1, right). Taking into account the compression of the granular material
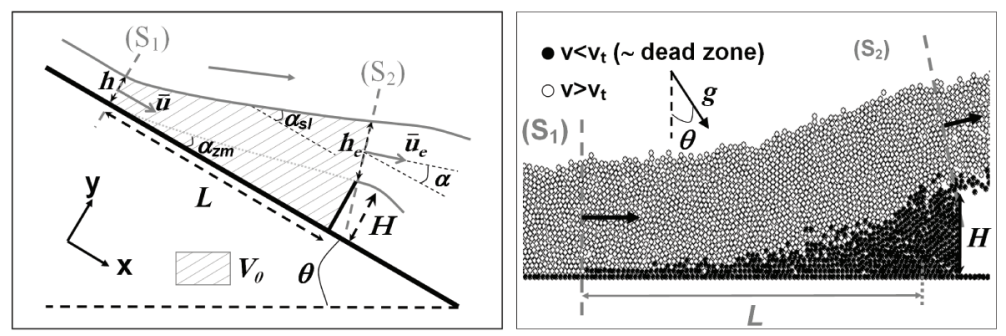

Figure 1: (left) Sketch of the granular stream overflowing the wall-like obstacle and (right) image showing a discrete numerical simulation. 
inside this dead zone, the product $\rho_{0} V_{0}$ is:

$$
\bar{\rho}_{0} V_{0}=\bar{\rho}\left(V_{0}-\frac{1}{2} L H\right)+\frac{1}{2} \rho_{z m} L H,
$$

with

$$
\begin{aligned}
V_{0}= & \frac{1}{2} h L\left[2+\tan \left(2 \alpha^{0}+\arctan \frac{H}{L}\right)\left(\frac{L}{h}-\delta_{h} \sin \alpha^{0}\right)\right. \\
& \left.+\frac{\delta_{h}}{L}(H-h) \sin \alpha^{0}\right],
\end{aligned}
$$

where $\rho_{z m}$ is the mean volume fraction inside the dead zone (a priori greater than the density of the fluid volume above the dead zone assumed to be equal to the density of the incoming flow $\bar{\rho}$ ), $L$ is the dead zone length assumed to be triangular in shape, $\delta_{h}=h_{e} / h$ is the depth ratio between sections $S_{2}$ and $S_{1}$ and $H$ is the wall height.

Closure equations are needed to define the geometric $\left(\alpha^{0}, L\right)$, hydrodynamic $\left(\delta_{u}=\bar{u}_{e} / \bar{u}, \delta_{h}=h_{e} / h\right)$ and rheological $\left(\bar{\mu}_{z m}\right)$ parameters. These closure equations are discussed in detail in [6] and will be briefly reminded in section 4.1 when calibrating the model on DEM simulations for steady-state flows. The equation giving the normal force $F_{N}$ on the obstacle will be compared to results from discrete numerical simulations in section 4.2.1.

\subsection{Unsteady flows}

Similar notation is adopted for unsteady flows. The momentum conservation in the control volume $V_{0}$ is:

$$
\frac{d}{d t}\left(\iint_{V_{0}} \rho \vec{u} d V\right)+\oint_{S} \rho \vec{u}(\vec{u} \cdot \vec{n}) d S=\oint_{S} p \vec{n} d S+\oint_{S} \sigma \cdot \vec{n} d S+\iint_{V_{0}} \vec{g} d V
$$

The relation $\bar{u}=0$ inside the dead zone leads to:

$$
\frac{d}{d t}\left(\iint_{V_{0}} \rho \vec{u} d V\right) \cdot \vec{x} \approx-\frac{d}{d t}\left(\iint_{V_{0}-\frac{1}{2} H L} \rho u d V\right) \approx-\frac{1}{2} \frac{d}{d t}\left(\bar{\rho} \bar{u}\left(1+\delta_{u}\right)\right)
$$

The last term is obtained by assuming that the mean density inside the fluid volume above the dead zone (equal to $V_{0}-1 / 2 H L$ ) is close to the incoming flow density $\bar{\rho}$ and by approximating the mean velocity with $\left(\bar{u}+\bar{u}_{e}\right) / 2=$ $\bar{u}\left(1+\delta_{u}\right) / 2$. In unsteady flow regime, a mass exchange between the granular material overflowing the obstacle and the granular material trapped upstream the obstacle is likely to occur (in particular at short times when the dead zone length increases): $\bar{\rho}_{e} h_{e} \bar{u}_{e}=\bar{\rho} h \bar{u}-d Q_{m}$, where $d Q_{m}$ corresponds to the mass exchange. If we assume that the mass exchange remains weak in comparison with incoming and outcoming mass flow rates, the mass conservation is still valid: $\bar{\rho}_{e} h_{e} \overline{u_{e}} \approx \bar{\rho} h \bar{u}$. 
This latter coupled with momentum conservation, and with $F_{T}(t) \sim 0$, gives the normal force:

$$
\begin{gathered}
F_{N}=F_{d}^{N}(t)+F_{h}^{N}(t)+F_{w}^{N}(t)-\bar{\mu}_{z m}(t)\left[F_{w}^{T}(t)+F_{d}^{T}(t)\right]+F_{m v}(t) \\
F_{d}^{N}(t)=\beta \bar{\rho}(t)[\bar{u}(t)]^{2} h(t)\left[1-\delta_{u}(t) \cos \alpha(t)\right] \\
F_{h}^{N}(t)=\frac{1}{2} k \bar{\rho}(t) g[h(t)]^{2} \cos \theta \\
F_{w}^{N}(t)=\bar{\rho}_{0}(t) V_{0}(t) g \sin \theta \\
F_{w}^{T}(t)=\bar{\rho}_{0}(t) V_{0}(t) g \cos \theta \\
F_{d}^{T}(t)=-\beta \bar{\rho}(t)[\bar{u}(t)]^{2} h(t) \delta_{u}(t) \sin \alpha(t) \\
F_{m v}(t)=-\frac{1}{2} \frac{d}{d t}\left[\bar{\rho}(t) \bar{u}(t)\left(1+\delta_{u}(t)\right)\right]
\end{gathered}
$$

$F_{m v}(t)$ is the force caused by the momentum variation over time. Closure equations are needed to define the geometric $\left[\alpha(t), L(t), V_{0}(t)\right]$, hydrodynamic $\left[\delta_{u}(t)=\bar{u}_{e}(t) / \bar{u}(t), \delta_{h}(t)=h_{e}(t) / h(t)\right]$ and rheological $\left[\bar{\mu}_{z m}(t)\right]$ parameters which depend on time $t$. These closure equations, discussed in detail in [2], will be briefly reminded in section 4.1 when calibrating the model on DEM simulations for avalanche flows. The equation giving the transient force $F_{N}(t)$ will be compared to results from DEM simulations in section 4.2.2.

\section{Discrete numerical simulations}

\subsection{Simulation method}

We performed numerical tests with a commercial code (PFC-Itasca) based on the molecular-dynamics method [3]. The method was shown to be successful to simulate dense granular flows $[4,5,9]$. The particles were assumed to interact through a damped linear spring for the normal force and a linear spring restricted to a threshold value according to friction between grains (Coulomb condition for the tangential force). Four microscopic parameters are needed: the normal stiffness $k_{n}$ ( $=10^{4} \mathrm{~N} \mathrm{~m}^{-1}$ in our simulations), the tangential stiffness $k_{t}\left(=1 / 2 k_{n}\right)$, the local particle friction $\mu(=0.5)$, and the damping coefficient related to the restitution coefficient $e(=0.5)$. Further details on the numerical method and the choice of the values given to the microscopic parameters are presented elsewhere $[1,2,6]$.

\subsection{Simulated systems}

The simulated systems consisted of an inclined slope and an upstream reservoir (Fig. 2). The grains in motion were spheres with a slight polydispersity $( \pm 10 \%$ in size), of mean diameter $d=1 \mathrm{~mm}$ and of density $\rho_{P}=2450 \mathrm{~kg} \mathrm{~m}^{3}$ (glass material). The bottom roughness was made with grains with the same properties as the grains in motion. The granular mass was released from the reservoir with an aperture 

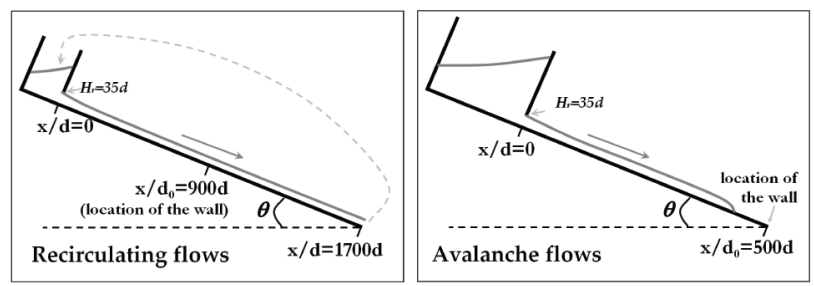

Figure 2: Simulated systems: steady (left) and avalanche (right) flows.

of constant height $H_{r}=35 d$. Two kinds of flows were investigated: steady recirculating flows [6] and time-varying avalanche flows [1, 2]. General features were different for each kind of flows: see details in Fig. 2. Flows with no obstacle, in a large range of slope inclinations $\left(16^{\circ} \leqslant \theta \leqslant 32^{\circ}\right)$, were first investigated. We characterized the changes over time in flow depth $h$, depth-averaged velocity $\bar{u}$ and volume fraction $\bar{\phi}$ at various locations, $x / d$, from the reservoir. Second, we measured the force from the granular stream on an obstacle located at the position $x_{0} / d$ (900 for recirculating flows and 500 for avalanche flows). The obstacle is a wall of height $H$ normal to the incoming flow and normal to the bottom. $H$ was systematically set to be equal to $h$ for steady recirculating flows and to the maximum in flow depth $h_{\max }$ for finite-sized avalanche flows.

\subsection{Force fluctuations and time-averaged force}

The main feature of the flow and obstacle interaction is the formation of a stagnant zone upstream the wall-like obstacle due to geometric trapping. The stagnant zone, or so called dead zone, is defined as a zone for which the mean velocity is smaller than a threshold velocity (Fig. 1, right) and is roughly triangular in shape, corresponding to the assumption made to derive the model presented in section 2. An intermittent force chains network caused by the discrete nature of the granular material is observed inside the dead zone leading to large fluctuations
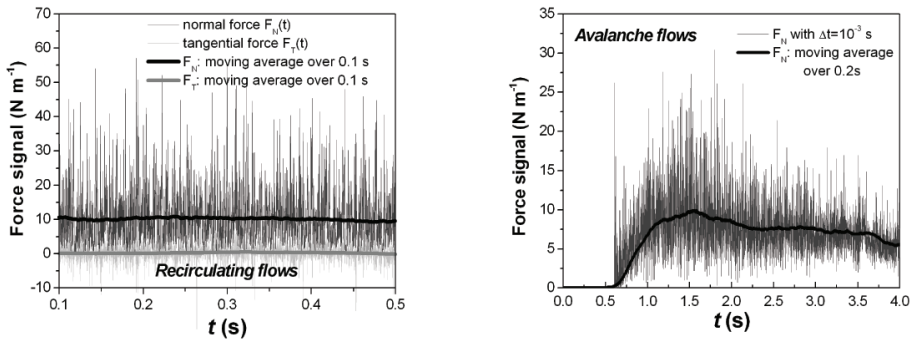

Figure 3: Typical force signals: (left) recirculating flow regime $\left(\theta=28^{\circ}\right)$ and (right) avalanche flow regime $\left(\theta=20^{\circ}\right)$. 
at high frequency in force signals (see in Fig. 3). We calculated the time-averaged force as shown by the thick continuous lines in Fig. 3. This force, corresponding to the time-averaged behavior of the discrete assembly, will be compared to the force derive from the hydrodynamic continuum model.

\section{Continuum model calibration and force prediction}

\subsection{Calibration of the closure equations}

In the steady flows' simulations, we measured the free-surface angle $\alpha_{s l}^{0}$, the depth ratio $\delta_{h}$, the dead zone length $L$ and the basal friction $\mu_{z m}$, which led to the following empirical closure equations: (i) $\alpha_{s l}^{0}=a \theta+b$ where $a$ and $b$ are constant parameters depending on typical friction angles characterizing the granular material (see details in [6]), (ii) $\delta_{u}=1-\kappa \alpha^{0}=1 / \delta_{h}$ (mass conservation) where $\kappa=(1-e) /(\pi / 2)$ with $e$ the restitution coefficient, (iii) $L=H / \tan \left(\theta-\theta_{\text {min }}\right)$ corresponding to the fact that the mean angle of the dead
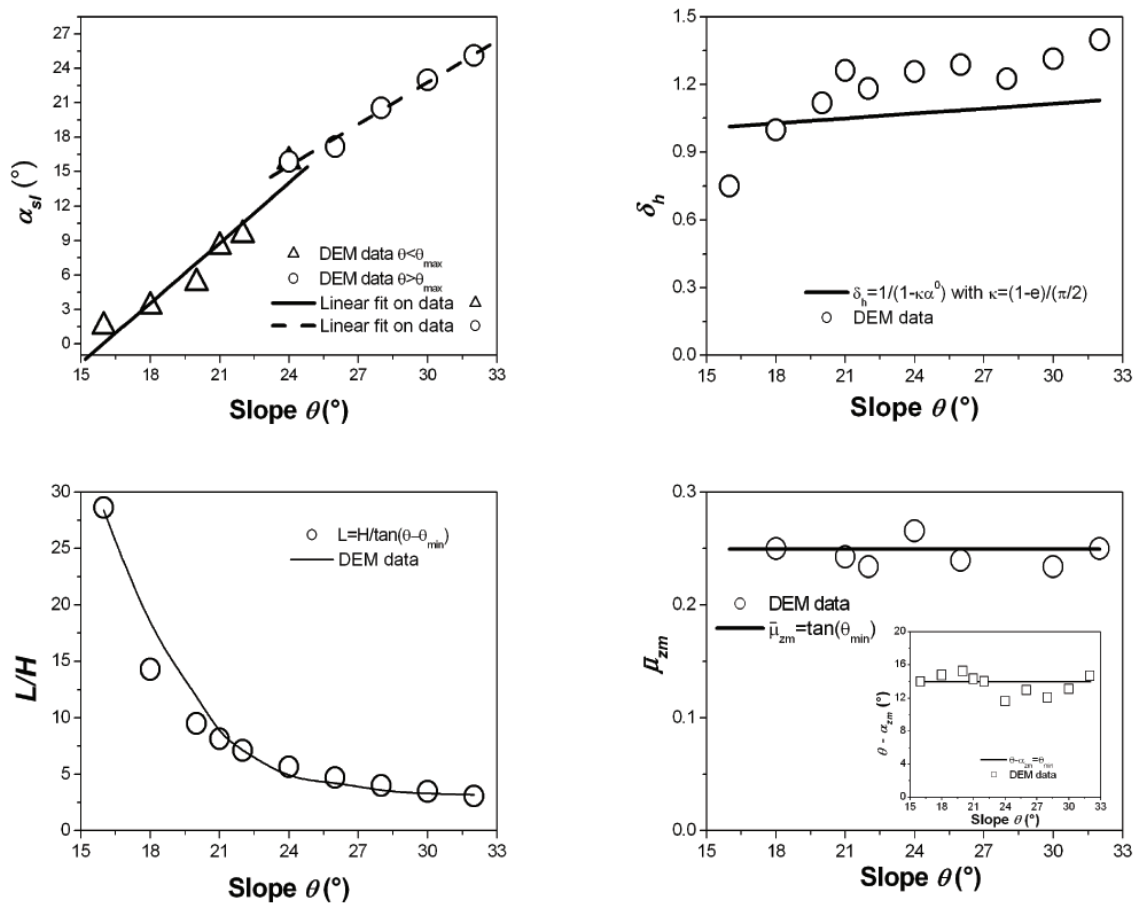

Figure 4: Model calibration in steady regime: (left-top) free-surface angle $\alpha_{s l}^{0}$; (right-top) flow depth-ratio $\delta_{h}$; (left-bottom) dead zone angle length $L / H$; (right-bottom) space-averaged friction $\bar{\mu}_{z m}$ (in inset is given the angle $\left.\theta-\alpha_{z m}\right)$. 

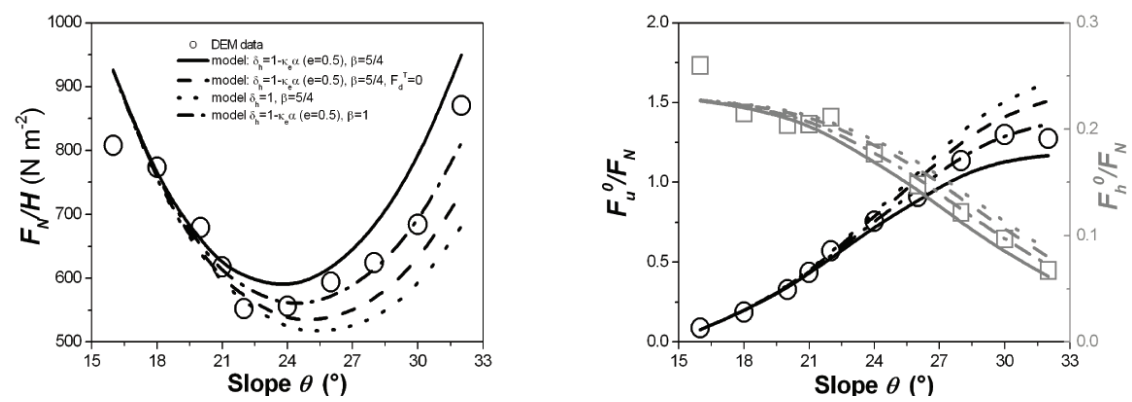

Figure 5: Pressure $F_{N} / H$ (left) and ratios $F_{u}^{0} / F_{N}$ and $F_{h}^{0} / F_{N}$ (right) versus the slope inclination $\theta$ with $F_{u}^{0}=1 / 2 \bar{\rho} \bar{u}^{2} h$ and $F_{h}^{0}=1 / 2 \bar{\rho} g h^{2} \cos \theta$.

zone with the horizontal was found to be constant and equal to $\theta_{\min }$ whatever the slope inclination $\theta$, and (iv) $\bar{\mu}_{z m}=\tan \theta_{\text {min }}$. Fig. 4 shows the prediction of these closure equations compared to discrete numerical data. Further detail of the calibration procedure are available in [6]. For time-varying avalanche flows, the angles $\alpha_{z m}(t)$ and $\alpha_{s l}(t)$ were assumed to decrease exponentially from the value $\pi / 2$ (no dead zone, $L \simeq 0$, at the time corresponding to the impact with the wall) to the value $\alpha_{z m}^{0}$ or $\alpha_{s l}^{0}$ corresponding to the steady regime. As a consequence, the deflection angle $\alpha$ decreases exponentially obeying the relation $\alpha(t)=\left[\alpha_{z m}(t)+\alpha_{s l}(t)\right] / 2$ and $L$ was derived thanks to $L(t)=H / \tan [\theta-$ $\alpha_{z m}(t)$. We measured the change over time of the dead zone length $L(t)$ which allows to validate these assumptions. The friction $\bar{\mu}_{z m}(t)$ was defined as it follows: $\bar{\mu}_{z m}(t)=\tan \left[\theta-\alpha_{z m}(t)\right]$. Further detail of calibration procedure can be found in [2].

\subsection{Force prediction compared to discrete numerical simulations}

\subsubsection{Steady recirculating flows}

In Fig. 5 is given the pressure $F_{N} / H$ versus the slope inclination in steady regime. The continuum model prediction (continuous line) is compared to numerical data. The continuum model slightly overestimates the data from discrete simulations for large slope inclinations. Model prediction is given for other assumptions: $F_{d}^{T}=0$ (dash line), $\delta_{h}=1$ instead of $\delta_{h}=1-\kappa \alpha$ with $\kappa=(1-e) /(\pi / 2)$ (dot line) and $\beta=1$ instead of $\beta=5 / 4$ (dash-dot line). The results show that it is needed to take into account the change in depth (and consequently in velocity) between sections $S_{1}$ and $S_{2}$ because $\delta_{h}=1$ gives bad results. Choosing $F_{d}^{T}=0$ leads to underestimate the data from discrete simulations. The model prediction can be improved by choosing $\beta=1$ rather than $\beta=5 / 4$.

\subsubsection{Time-varying avalanche flows}

In Fig. 6 is given the temporal force signal for various slopes $\theta=18^{\circ}$ (black), $22^{\circ}$ (dark grey) and $32^{\circ}$ (light grey). Three phases of the flow-wall interaction 

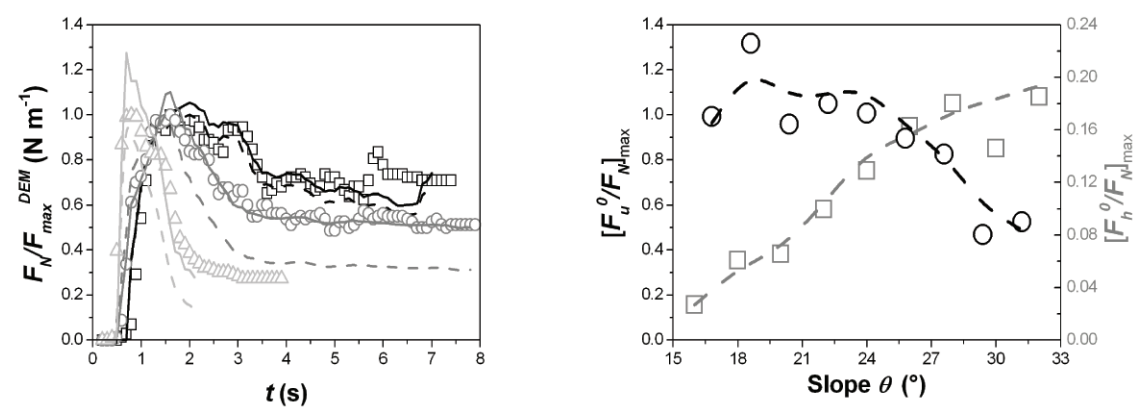

Figure 6: Avalanche force $F_{N}$ normalized by $F_{\max }^{D E M}$ versus $t$ for $\theta=18^{\circ}, 22^{\circ}$ and $32^{\circ}$ (left); ratios $\left[F_{u}^{0} / F_{N}\right]_{\max }$ and $\left[F_{h}^{0} / F_{N}\right]_{\max }$ versus $\theta$ (right). Comparison between DEM data (symbols) and continuum model (dash line and continuous line with $\mu_{z m}^{f i t}(t)=c_{\mu} \bar{\mu}_{z m}(t)$ ).

can be distinguished: (i) a fast increase in force (corresponding to the dead zone formation), (ii) a force peak and (iii) a slow decrease in force. The agreement between discrete numerical data (symbols) and the continuum model (dash line) is good for the first two phases (i) and (ii) whatever the slope inclination (see also Fig. 6, right, showing the maximum forces normalized by the typical hydrostatic and dynamic forces). For larger slope inclinations, a gap is observed for the third phase corresponding to the avalanche tail. The model largely underestimates the DEM data. This gap remains an open question. A fitting procedure consisting in considering a parameter $c_{\mu}$ defined by $\mu_{z m}^{f i t}(t)=c_{\mu} \bar{\mu}_{z m}(t)$ was proposed in [2]. It allows to reproduce the slow decrease in force during the interaction between the wall and the avalanche tail (continuous lines in Fig. 6, left). $c_{\mu}$ is a constant coefficient depending on the slope inclination. Let us note that considering $F_{d}^{T}=0$ does not influence the results in the transient avalanche regime investigated here.

\subsection{Various contribution to the total force}

he continuum model allows to quantify the various contributions to the total force: the dynamic contribution $F_{d}^{N}$, the hydrostatic contribution $F_{w}^{N}$, the weight $F_{w}^{T}$ of the volume disturbed by the presence of the wall (control volume), the basal friction force $F_{f}=-\bar{\mu}_{z m}\left[F_{w}^{T}+F_{d}^{T}\right]$ and the force $F_{m v}$ caused by momentum variation over time (equal to zero in steady regime). Fig. 7 shows the results for both steady recirculating flows and time-avarying avalanche flows.

\section{Discussion and conclusion: contribution to avalanche dam design}

The cross-comparison between the continuum model and the DEM data shows the ability of hydrodynamics approaches to mimic the macroscopic behavior of an 

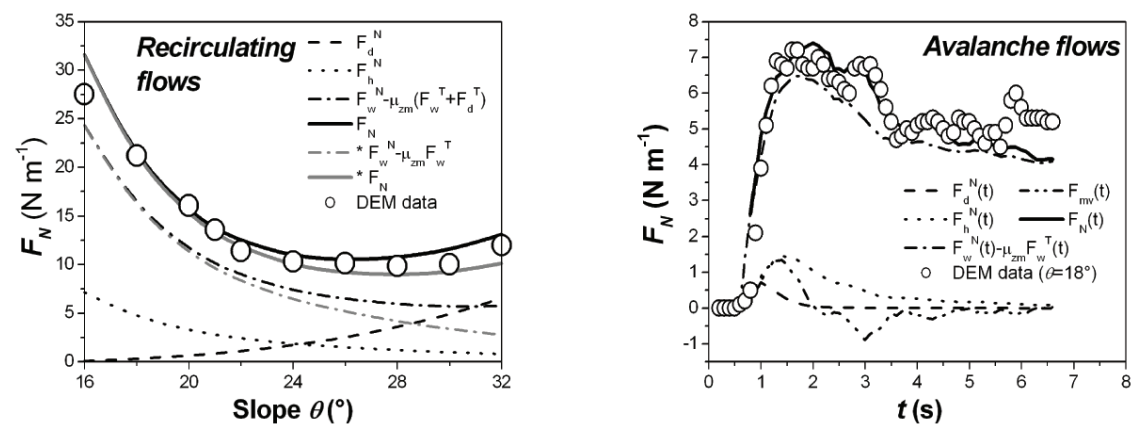

Figure 7: The various contributions to the total force: (left) steady and (right) avalanche flows (example for $\theta=18^{\circ}$ ).

assembly of discrete particles in term of the mean force exerted on a wall if the model closure equations (geometry, kinematics and rheology) are well calibrated. The model prediction is very good in steady regime and some improvement is still needed for the avalanche regime. An open fundamental question is the need of fitting the basal friction to reproduce the force after the peak at high slopes [2]. Nevertheless, the continuum model provides an analytical formulation to derive the hydrodynamic force on a wall taking into account the various contributions to the force. In the framework of relatively slow avalanches, the hydrostatic force of the incoming flow and the apparent weight of the fluid volume disturbed by the obstacle should be considered and can be derived from the continuum model. Engineers in charge of protection dams' design against granular snow avalanches often consider the following rule of thumb [8]: $F_{\text {design }}=C_{d} P_{d} S_{d a m}$ where $P_{d}=1 / 2 \rho u^{2}$ is the dynamic pressure, $S_{d a m}$ is the dam area prone to the avalanche and $C_{d}$ is a drag coefficient depending on snow type and obstacle shape. Our study clearly points at the shortcoming of the snow engineering classical approach. Attention should be also paid on the following points: (i) the influence of more complex material properties (cohesion, clustering) on the closure equations in order to apprehend more complex granular materials close to natural ones, (ii) the more complex flow and obstacle geometries with possible lateral overflows (see a preliminary attempt in [7]) and (iii) the fluid/structure coupling processes which can influence the force the obstacle is likely to undergo (the wall was fixed and rigid), particularly in the context of force fluctuations (see Fig. 3) which were not tackled within the time-average hydrodynamic approach presented in this paper.

\section{Acknowledgements}

This study received financial support from the following projects: ANR-MONHA, ANR-MOPERA and DYNAVAL (INTERREG-ALCOTRA). 


\section{References}

[1] B. Chanut, T. Faug, and M. Naaim. Mean force on a wall overflowed by dense granular avalanches: Discrete numerical simulations. In Powders and 2009: Proceedings of the 6th international conference on micromechanics of granular media, volume 1145, pages 609-612, 2009.

[2] B. Chanut, T. Faug, and M. Naaim. Time-varying force from dense granular avalanches on a wall. Physical Review E, 82(4):041302, 2010.

[3] P.A. Cundall and O.D.L. Strack. A discrete numerical model for granular assemblies. Geotechnique, 29:47-65, 1979.

[4] F. da Cruz, S. Emam, M. Prochnow, J-N. Roux, and F. Chevoir. Rheophysics of dense granular flows : discrete simulation of plane shear flows. Physical Review E, 72:021309, 2005.

[5] D. Ertas, G. S. Grest, T. C. Halsey, D. Levine, and L.E. Silbert. Gravity-driven dense granular flows. Europhysics Letters, 56(2):214, 2001.

[6] T. Faug, R. Beguin, and B. Chanut. Mean steady granular force on a wall overflowed by free-surface gravity-driven dense flows. Physical Review E, 80(2):021305, 2009.

[7] T. Faug, B. Chanut, R. Beguin, M. Naaim, M. Thibert, and D. Baroudi. A simple analytical model for pressure on obstacles induced by snow avalanches. Annals of Glaciology, 51(54):1-8, 2010.

[8] B. Salm, A. Burkard, and H. Gubler. Berechnung von fliesslawinen, eine anleitung für praktiker mit beispielen. Technical Report 47, Eidgenossisches Institut für Schnee und Lawinenforschung (Davos), 1990.

[9] L.E. Silbert, D. Ertas, G. S. Grest, T. C. Halsey, D. Levine, and S. J. Plimpton. Granular flow down an inclined plane: Bagnold scaling and rheology. Physical Review E, 64:051302, 2001. 